\title{
Inviscid limit for the compressible Euler system with non-local interactions
}

\author{
Jan Březina ${ }^{1}$ \\ Tokyo Institute of Technology, \\ 2-12-1 Ookayama, Meguro-ku, \\ Tokyo, 152-8550 \\ Japan \\ Václav Mácha ${ }^{2}$ \\ Industry-University Research Center, Yonsei University, \\ 50 Yonsei-ro Seodaemun-gu, \\ Seoul, 03722 \\ Republic of Korea
}

\begin{abstract}
The collective behavior of animals can be modeled by a system of equations of continuum mechanics endowed with extra terms describing repulsive and attractive forces between the individuals. This system can be viewed as a generalization of the compressible Euler equations with all of its unpleasant consequences, e.g., the non-uniqueness of solutions. In this paper, we analyze the equations describing a viscous approximation of a generalized compressible Euler system and we show that its dissipative measure-valued solutions tend to a strong solution of the Euler system as viscosity tends to 0, provided that the strong solution exists.

Keywords: Euler system, measure-valued solution, collective behavior, artificial viscosity
\end{abstract}

2010 MSC: 35Q31, 35D40, 35D99

\footnotetext{
${ }^{1}$ email:brezina@math.titech.ac.jp

2 email:macha@math.cas.cz

The research of V. M. has been supported by the grant NRF-20151009350.
}

Preprint submitted to Elsevier

July 16, 2018 


\section{Introduction}

Recently the modeling of collective behavior of animals has became a popular topic. In this paper we study the hydrodynamical model for collective behavior which can be obtained as a mean-field limit of the Cucker-Smale flocking model (see [1, 2]). As the hydrodynamical model is in fact an Euler-type system, its solutions may be constructed by means of convex integration (see [3, 4, 5 , 6]) and thus are in general non-unique and irregular even for smooth initial data. Then the question of choosing the unique physically reasonable solution comes into play (see [3]). The inviscid limit is often considered to be one of the criteria of admissibility for the Euler-type problems. In this paper we study the question whether a solution to the above mentioned Euler type system could be understood as a limit of viscous approximations.

More precisely, we prove that under certain smoothness conditions imposed on a solution to the Euler system (1.1), this solution can be understood as a limit of solutions to the approximate Navier-Stokes system (1.6).

We consider the following system:

$$
\begin{aligned}
\partial_{t} \rho+\operatorname{div}_{x}(\rho \mathbf{u})= & 0 \\
\partial_{t}(\rho \mathbf{u})+\operatorname{div}_{x}(\rho \mathbf{u} \otimes \mathbf{u})+\nabla_{x} p(\rho)= & \left(1-H\left(|\mathbf{u}|^{2}\right)\right) \rho \mathbf{u} \\
& -\rho \int_{\Omega} \nabla_{x} K(x-y) \rho(y) \mathrm{d} y \\
& +\rho \int_{\Omega} \psi(x-y) \rho(y)(\mathbf{u}(y)-\mathbf{u}(x)) \mathrm{d} y,
\end{aligned}
$$

where $\rho=\rho(t, x)$ and $\mathbf{u}=\mathbf{u}(t, x)$ denote the unknown density and velocity; $p=p(\rho)$ is the pressure; $H=H(z), z \in[0,+\infty)$ is 'friction'; $K=K(x)$ and $\psi=\psi(x)$ represent the non-local interaction forces acting on the medium.

For the sake of simplicity, we consider the spatially periodic boundary conditions, i.e.,

$$
\Omega=\left(\left.[-1,1]\right|_{-1,1}\right)^{N}, \quad N=2,3 .
$$


In accordance with [3] we assume that

$$
\begin{gathered}
p \in C([0, \infty)) \cap C^{2}(0, \infty), \quad p(0)=0, \quad p^{\prime}(\rho)>0 \text { whenever } \rho>0, \\
\liminf _{\rho \rightarrow \infty} p^{\prime}(\rho)>0, \quad \liminf _{\rho \rightarrow \infty} \frac{P(\rho)}{p(\rho)}>0,
\end{gathered}
$$

where

$$
P(\rho)=\rho \int_{1}^{\rho} \frac{p(z)}{z^{2}} \mathrm{~d} z
$$

together with

$$
H \in C^{2}([0, \infty)), \quad 0 \leq H(z) \leq H_{\infty}:=\lim _{z \rightarrow \infty} H(z), \quad H^{\prime}(z) \geq 0 \text { for all } z \geq z_{0}
$$

and

$$
K \in C^{2}(\Omega), \quad \psi \in C^{1}(\Omega), \quad \psi \geq 0,
$$

$K$ and $\psi$ symmetric, i.e., $K(x)=K(-x), \psi(x)=\psi(-x)$.

Note that we could take $H, K$ and $\psi$ so that no extra terms appear on the right-hand side of (1.1) 2 . Therefore, our result also covers the case of compressible Euler equations. Finally, the system is endowed with the following initial conditions:

$$
\rho(0)=\rho_{0}, \quad \mathbf{u}(0)=\mathbf{u}_{0}
$$

We show that any sufficiently smooth solution to (1.1) can be obtained as a limit of dissipative measure-valued solutions to the viscous approximation

$$
\begin{aligned}
& \partial_{t} \rho^{\varepsilon}+\operatorname{div}_{x}\left(\rho^{\varepsilon} \mathbf{u}^{\varepsilon}\right)=0, \\
& \partial_{t}\left(\rho^{\varepsilon} \mathbf{u}^{\varepsilon}\right)+\operatorname{div}_{x}\left(\rho^{\varepsilon} \mathbf{u}^{\varepsilon} \otimes \mathbf{u}^{\varepsilon}\right)+\nabla_{x} p\left(\rho^{\varepsilon}\right)=\varepsilon \Delta_{x} \mathbf{u}^{\varepsilon}+\left(1-H\left(\left|\mathbf{u}^{\varepsilon}\right|^{2}\right)\right) \rho^{\varepsilon} \mathbf{u}^{\varepsilon} \\
& -\rho^{\varepsilon} \int_{\Omega} \nabla_{x} K(x-y) \rho^{\varepsilon}(y) \mathrm{d} y \\
& +\rho^{\varepsilon} \int_{\Omega} \psi(x-y) \rho^{\varepsilon}(y)\left(\mathbf{u}^{\varepsilon}(y)-\mathbf{u}^{\varepsilon}(x)\right) \mathrm{d} y,
\end{aligned}
$$

on $\Omega$ as $\varepsilon$ tends to zero. The unknowns $\rho_{\varepsilon}$ and $\mathbf{u}_{\varepsilon}$ are assumed to satisfy the initial conditions

$$
\rho^{\varepsilon}(0)=\rho_{0}^{\varepsilon}, \quad \mathbf{u}^{\varepsilon}(0)=\mathbf{u}_{0}^{\varepsilon},
$$


such that $\rho_{0}^{\varepsilon} \rightarrow \rho_{0}$ and $\mathbf{u}_{0}^{\varepsilon} \rightarrow \mathbf{u}_{0}$ in a sense specified later on.

As there are two crucial non-linearities in (1.6) 2 , namely $p\left(\rho^{\varepsilon}\right)$ and $(1-$ $\left.H\left(\left|\mathbf{u}^{\varepsilon}\right|^{2}\right)\right) \rho^{\varepsilon} \mathbf{u}^{\varepsilon}$, it seems that the existence of weak solutions to (1.6) cannot be established by the currently available theory. Thus we turn our attention towards the measure-valued solutions. Motivated by [7] we define a measurevalued solution to (1.6) and (1.7) in the following way.

Definition 1.1. Let $\varepsilon>0, T>0$ and $Q_{\tau}:=(0, \tau) \times \Omega$ for every $\tau \in[0, T]$. We say that a parametrized measure $\left\{\nu_{t, x}\right\}_{(t, x) \in Q_{T}}$,

$$
\nu \in L_{w *}^{\infty}\left(Q_{T}, \mathcal{P}\left([0, \infty) \times \mathbb{R}^{N}\right), \rho(t, x):=\left\langle\nu_{t, x}, s\right\rangle, \mathbf{u}(t, x):=\left\langle\nu_{t, x}, \mathbf{v}\right\rangle, 3\right.
$$

is a dissipative measure-valued solution to (1.6) in $Q_{T}$ and (1.7) in $\Omega$ with the dissipation defect $\mathcal{D} \in L^{\infty}(0, T), \mathcal{D} \geq 0$ if:

- For every $\psi \in C^{1}([0, T] \times \Omega)$, the equation of continuity

$$
\begin{aligned}
\int_{\Omega} \rho(\tau) \varphi(\tau) \mathrm{d} x & -\int_{Q_{\tau}} \rho \partial_{t} \varphi \mathrm{d} x \mathrm{~d} t \\
& -\int_{Q_{\tau}}\left\langle\nu_{t, x}, s \mathbf{v}\right\rangle \nabla_{x} \varphi(t, x) \mathrm{d} x \mathrm{~d} t=\int_{\Omega}\left\langle\nu_{0 x}, s\right\rangle \varphi(0, x) \mathrm{d} x,
\end{aligned}
$$

holds for all $\tau \in[0, T]$.

- There exists a measure $\mu \in L^{\infty}(0, T, \mathcal{M}(\Omega))$ and a constant $c>0$ such that for every $\varphi \in C^{1}([0, T] \times \Omega)^{N}$,

$$
\left|\int_{\Omega} \varphi(\tau) \mathrm{d} \mu(\tau)\right| \leq c \mathcal{D}(\tau)\|\varphi(\tau)\|_{L^{\infty}(\Omega)},
$$

\footnotetext{
${ }^{3}$ Hereinafter we use convention $\left\langle\nu_{t, x}, F(s, \mathbf{v})\right\rangle=\int_{[0, \infty) \times \mathbb{R}^{N}} F(s, \mathbf{v}) \mathrm{d} \nu_{\mathrm{t}, \mathrm{x}}$ where $s$ and $\mathbf{v}$ represent the dummy variables for $\rho$ and $\mathbf{u}$, respectively.
} 
and the momentum equation

$$
\begin{gathered}
\int_{\Omega}\left\langle\nu_{\tau, x}, s \mathbf{v}\right\rangle \boldsymbol{\varphi}(\tau, x) \mathrm{d} x-\int_{Q_{\tau}}\left\langle\nu_{t, x}, s \mathbf{v}\right\rangle \partial_{t} \boldsymbol{\varphi}(t, x) \mathrm{d} x \mathrm{~d} t \\
-\int_{Q_{\tau}}\left\langle\nu_{t, x}, s \mathbf{v} \otimes \mathbf{v}\right\rangle \nabla_{x} \boldsymbol{\varphi}(t, x) \mathrm{d} x \mathrm{~d} t+\varepsilon \int_{Q_{\tau}} \nabla_{x} \mathbf{u}: \nabla_{x} \boldsymbol{\varphi} \mathrm{d} x \mathrm{~d} t \\
\quad-\int_{Q_{\tau}}\left\langle\nu_{t, x}, p(s)\right\rangle \operatorname{div}_{x} \varphi(t, x) \mathrm{d} x \mathrm{~d} t \\
=\int_{Q_{\tau}}\left\langle\nu_{t, x},\left(1-H\left(|\mathbf{v}|^{2}\right)\right) s \mathbf{v}\right\rangle \boldsymbol{\varphi}(t, x) \mathrm{d} x \mathrm{~d} t \\
-\int_{Q_{\tau}} \rho(t, x) \int_{\Omega} \nabla_{x} K(x-y) \rho(t, y) \mathrm{d} y \boldsymbol{\varphi}(t, x) \mathrm{d} x \mathrm{~d} t \\
+\int_{Q_{\tau}} \rho(t, x) \int_{\Omega} \psi(x-y)\left\langle\nu_{t, y}, s \mathbf{v}\right\rangle \mathrm{d} y \boldsymbol{\varphi}(t, x) \mathrm{d} x \mathrm{~d} t \\
-\int_{Q_{\tau}}\left\langle\nu_{t, x}, s \mathbf{v}\right\rangle \int_{\Omega} \psi(x-y) \rho(t, y) \mathrm{d} y \boldsymbol{\varphi}(t, x) \mathrm{d} x \mathrm{~d} t \\
+\int_{\Omega}\left\langle\nu_{0 x}, s \mathbf{v}\right\rangle \boldsymbol{\varphi}(0, x) \mathrm{d} x+\int_{0}^{\tau} \int_{\Omega} \nabla_{x} \boldsymbol{\varphi}(t) \mathrm{d} \mu(t) \mathrm{d} t
\end{gathered}
$$

hold for all $\tau \in[0, T]$.

- The energy inequality

$$
\begin{gathered}
\int_{\Omega}\left\langle\nu_{\tau, x}, \frac{1}{2} s|\mathbf{v}|^{2}+P(s)\right\rangle \mathrm{d} x+\frac{1}{2} \int_{\Omega}(\rho K * \rho)(\tau) \mathrm{d} x \\
+\varepsilon \int_{Q_{\tau}}\left|\nabla_{x} \mathbf{u}\right|^{2} \mathrm{~d} x \mathrm{~d} t+\mathcal{D}(\tau) \\
\leq \int_{\Omega}\left\langle\nu_{0 x}, \frac{1}{2} s|\mathbf{v}|^{2}+P(s)+\frac{1}{2} s K *\left\langle\nu_{0 y}, s\right\rangle\right\rangle \mathrm{d} x \\
+\int_{Q_{\tau}}\left\langle\nu_{t, x},\left(1-H\left(|\mathbf{v}|^{2}\right)\right) s|\mathbf{v}|^{2}\right\rangle \mathrm{d} x \mathrm{~d} t+c \int_{0}^{\tau} \mathcal{D}(t) \mathrm{d} t \\
-\int_{Q_{\tau}} \int_{\Omega}\left(\left\langle\nu_{t, x}, s|\mathbf{v}|^{2}\right\rangle \rho(t, y)-\left\langle\nu_{t, x}, s \mathbf{v}\right\rangle\left\langle\nu_{t, y}, s \mathbf{v}\right\rangle\right) \psi(x-y) \mathrm{d} y \mathrm{~d} x \mathrm{~d} t
\end{gathered}
$$

holds for a.a. $\tau \in(0, T)$.

With $\nu_{0 x}=\delta_{\left(\rho_{0}(x), \mathbf{u}_{0}(x)\right)}$ for a.a. $x \in \Omega$ and $c$ independent of $\varepsilon$ and any solution. 
Remark 1.2. It is not difficult to see that

$$
\begin{gathered}
\int_{\Omega} \int_{\Omega}\left(\left\langle\nu_{t, x}, s|\mathbf{v}|^{2}\right\rangle \rho(t, y)-\left\langle\nu_{t, x}, s \mathbf{v}\right\rangle\left\langle\nu_{t, y}, s \mathbf{v}\right\rangle\right) \psi(x-y) \mathrm{d} y \mathrm{~d} x \\
=\int_{\Omega} \int_{\Omega}\left(\frac{1}{2}\left\langle\nu_{t, x}, s|\mathbf{v}|^{2}\right\rangle \rho(t, y)-\left\langle\nu_{t, x}, s \mathbf{v}\right\rangle\left\langle\nu_{t, y}, s \mathbf{v}\right\rangle\right. \\
\left.\quad+\frac{1}{2} \rho(t, x)\left\langle\nu_{t, y}, s|\mathbf{v}|^{2}\right\rangle\right) \psi(x-y) \mathrm{d} y \mathrm{~d} x=: \int_{\Omega} \int_{\Omega} \mathcal{I} \mathrm{d} y \mathrm{~d} x
\end{gathered}
$$

is positive by the use of the Fubini lemma,

$$
\begin{array}{r}
\mathcal{I}=\psi(x-y)\left(\frac{1}{2} \int s|\mathbf{v}|^{2} \mathrm{~d} \nu_{t, x}(s, \mathbf{v}) \int \sigma \mathrm{d} \nu_{t, y}(\sigma, \mathbf{w})\right. \\
\left.-\int s \mathbf{v} \mathrm{d} \nu_{t, x}(s, v) \int \sigma \mathbf{w} \mathrm{d} \nu_{t, y}(\sigma, \mathbf{w})+\frac{1}{2} \int s \mathrm{~d} \nu_{t, x}(s, \mathbf{v}) \int \sigma \mathbf{w}^{2} \mathrm{~d} \nu_{t, y}(\sigma, \mathbf{w})\right) \\
=\frac{1}{2} \iint s \sigma|\mathbf{v}-\mathbf{w}|^{2} \mathrm{~d} \nu_{t, x}(s, \mathbf{v}) \mathrm{d} \nu_{t, y}(\sigma, \mathbf{w}) \geq 0
\end{array}
$$

Here, the integration is over $[0, \infty) \times \mathbb{R}^{N}$.

In this paper we show that the concept of dissipative measure-valued solution is an appropriate one for our problem. On the one hand it is general enough to allow for existence, otherwise unavailable for weak solutions, and for another it is robust enough to provide an inviscid limit. The existence of measure-valued solutions is given as follows.

Theorem 1.3. Let $\varepsilon>0$ and $\rho_{0}^{\varepsilon}, \mathbf{u}_{0}^{\varepsilon}$ be initial data with a finite energy, i.e.,

$$
\int_{\Omega}\left(\frac{1}{2} \rho_{0}^{\varepsilon}\left|\mathbf{u}_{0}^{\varepsilon}\right|^{2}+P\left(\rho_{0}^{\varepsilon}\right)\right) d x<\infty
$$

If the hypotheses (1.2), (1.3) and (1.4) are satisfied, then for any $T>0$ there exists a dissipative measure-valued solution to (1.6) and (1.7) in the sense of Definition 1.1.

As already mentioned, we consider the dissipative measure-valued solutions to accommodate for the nonlinearities in $(1.6)_{2}$. We would like to emphasize that when $p(\rho) \sim \rho^{\gamma}, \gamma>\frac{3}{2}$ and $H(z) \equiv$ const. one can obtain the existence of weak solutions in a standard manner (cf. [8]).

Before stating the main result we would like to introduce the so-called relative entropy functional. Let $\nu: \Omega \rightarrow \mathcal{P}\left([0, \infty) \times \mathbb{R}^{N}\right)$ be a Young measure and let 
$r: \Omega \mapsto(0, \infty)$ and $\mathbf{U}: \Omega \mapsto \mathbb{R}^{N}$ be smooth functions. We then define the relative entropy functional $\mathcal{E}$ as

$\mathcal{E}(\nu, r, \mathbf{U}):=\int_{\Omega}\left\langle\nu_{x}, \frac{1}{2} s|\mathbf{v}-\mathbf{U}(x)|^{2}+P(s)-P^{\prime}(r(x))(s-r(x))-P(r(x))\right\rangle \mathrm{d} x$.

Theorem 1.4. Let $T>0$ and the hypotheses (1.2), (1.3) and (1.4) are satisfied. Let $\left\{\left(\nu^{\varepsilon}, D^{\varepsilon}\right)\right\}_{\varepsilon>0}$ be a family of dissipative measure-valued solutions to (1.6) emanating from the initial conditions (1.7). Let $(r, \mathbf{U})$ be a strong solution to (1.1) in $Q_{T}$ endowed with (1.5) such that $r \geq c>0$ for some c. Moreover, assume that

$$
\left(\rho_{0}^{\varepsilon}, \mathbf{u}_{0}^{\varepsilon}\right) \rightarrow\left(r_{0}, \mathbf{U}_{0}\right)
$$

in the following sense:

$$
\mathcal{E}\left(\nu_{0}^{\varepsilon}, r_{0}, \mathbf{U}_{0}\right) \rightarrow 0,
$$

where $\nu_{0 x}^{\varepsilon}=\delta_{\left(\rho_{0}^{\varepsilon}(x), \mathbf{u}_{0}^{\varepsilon}(x)\right)}$ for a.a $x \in \Omega$.

Then $\left\{\left(\nu^{\varepsilon}, D^{\varepsilon}\right)\right\}_{\varepsilon>0}$ tends to $(r, \mathbf{U})$ in the following sense:

$$
\underset{\tau \in(0, T)}{\operatorname{ess} \sup } \mathcal{E}\left(\nu_{\tau, \cdot}^{\varepsilon}, r(\tau), \mathbf{U}(\tau)\right) \rightarrow 0 \quad \text { and } \quad \underset{\tau \in(0, T)}{\text { ess } \sup } \mathcal{D}^{\varepsilon}(\tau) \rightarrow 0,
$$

as $\varepsilon \rightarrow 0$.

Remark 1.5. Due to the assumptions there exist $\underline{r}, \bar{r}>0$ such that $\underline{r} \leq \frac{r(t, x)}{2} \leq$ $2 r(t, x) \leq \bar{r}$ for all $(t, x) \in Q_{T}$. According to [9, (4.1)] we have

$$
\begin{gathered}
\int_{(0, \infty) \times \mathbb{R}^{N}}|s-r(\tau, x)| \mathrm{d} \nu_{\tau, x}^{\varepsilon}(s, \mathbf{v}) \leq\left(\int_{[\underline{r}, \bar{r}] \times \mathbb{R}^{N}}|s-r(\tau, x)|^{2} \mathrm{~d} \nu_{\tau, x}^{\varepsilon}(s, \mathbf{v})\right)^{\frac{1}{2}} \\
+c\left[\left(\int_{(\bar{r}, \infty) \times \mathbb{R}^{N}} s \mathrm{~d} \nu_{\tau, x}^{\varepsilon}(s, \mathbf{v})\right)^{\frac{1}{2}}+\left(\int_{(0, \underline{r}) \times \mathbb{R}^{N}} 1 \mathrm{~d} \nu_{\tau, x}^{\varepsilon}(s, \mathbf{v})\right)^{\frac{1}{2}}\right]^{2} \\
\leq c\left\langle\nu_{\tau, x}^{\varepsilon}, P(s)-P^{\prime}(r(\tau, x))(s-r(\tau, x))-P(r(\tau, x))\right\rangle^{\frac{1}{2}}\left(1+\rho^{\varepsilon}(\tau, x)\right)^{\frac{1}{2}},
\end{gathered}
$$

and thus by Hölder inequality we get

$$
\begin{aligned}
\left\|\left(\rho^{\varepsilon}-r\right)(\tau)\right\|_{L^{1}(\Omega)} \leq \int_{\Omega}\left\langle\nu_{\tau, x}^{\varepsilon},|s-r(\tau, x)|\right\rangle \mathrm{d} x & \\
\leq \int_{\Omega}\left\langle\nu_{\tau, x}^{\varepsilon}, P(s)-P^{\prime}(r(\tau, x))(s-r(\tau, x))-\right. & P(r(\tau, x))\rangle^{\frac{1}{2}}\left(1+\rho^{\varepsilon}(\tau, x)\right)^{\frac{1}{2}} \mathrm{~d} x \\
& \leq c \mathcal{E}\left(\nu_{\tau, \cdot}^{\varepsilon}, r(\tau), \mathbf{U}(\tau)\right)^{\frac{1}{2}}, \quad(1.10)
\end{aligned}
$$


where we use the fact that $\int_{\Omega} \rho^{\varepsilon}(\tau) \mathrm{d} x=\int_{\Omega} \rho_{0}^{\varepsilon} \mathrm{d} x$ for all $\tau \in[0, T]$ and $\left\|\rho_{0}^{\varepsilon}\right\|_{L^{1}(\Omega)} \leq$ $c$ for some $c>0$ independent of $\varepsilon$ and any solution.

Thus (1.9) implies that

$$
\rho^{\varepsilon} \rightarrow r \quad \text { in } L^{\infty}\left(0, T, L^{1}(\Omega)\right)
$$

as $\varepsilon \rightarrow 0$.

It is worth pointing out that the result of Theorem 1.4 applies also to suitable weak solutions (see [9]) as every suitable weak solution can be seen as a dissipative measure-valued solution.

The rest of this paper is devoted to the proofs of Theorems 1.3 and 1.4 In Section 2 we show the existence of dissipative measure-valued solutions. Section [3] is concerned with relative entropy inequality for the dissipative measure-valued solutions. Finally, this is used in Section 4 to prove Theorem 1.4 .

\section{Existence of measure-valued solutions}

Throughout this section let $T>0$ and $\varepsilon>0$ be fixed and we omit the index $\varepsilon$ for the sake of clarity. We construct a measure-valued solution to (1.6) and (1.7) by means of a two-level approximation. First, we introduce a system with viscous penalization of the density. Second, we solve it by the Faedo-Galerkin approximation. However, as we would like to proceed in both limits at the same time, we take the coefficient of artificial viscosity dependent on $n$ - the coefficient of the Faedo-Galerkin approximation.

\subsection{Faedo-Galerkin approximation}

We consider a family of nested finite dimensional spaces $X_{n}, n=1,2, \ldots$ consisting of smooth vector-valued functions defined on $\Omega$.

The equation of continuity (1.6) 1 is regularized using vanishing artificial viscosity as

$$
\begin{aligned}
\partial_{t} \rho^{n}+\operatorname{div}_{x}\left(\rho^{n} \mathbf{u}^{n}\right) & =\frac{1}{n} \Delta_{x} \rho^{n} & & \text { in } Q_{T}, \\
\rho^{n}(0) & =\rho_{0}^{n} & & \text { in } \Omega .
\end{aligned}
$$


We continue in the spirit of [8, Chapter 7] and we look for the Faedo-Galerkin approximate solutions, namely, we look for velocities $\mathbf{u}^{n} \in C\left([0, T] ; X_{n}\right)$ that satisfy the integral identity

$$
\begin{aligned}
& \int_{\Omega} \rho^{n} \mathbf{u}^{n}(\tau) \cdot \boldsymbol{\eta} \mathrm{d} x-\int_{\Omega} \mathbf{m}_{0}^{n} \cdot \boldsymbol{\eta} \mathrm{d} x \\
& =\int_{Q_{\tau}}\left(\rho^{n} \mathbf{u}^{n} \otimes \mathbf{u}^{n}-\varepsilon \nabla_{x} \mathbf{u}^{n}\right): \nabla_{x} \boldsymbol{\eta}+p\left(\rho^{n}\right) \operatorname{div}_{x} \boldsymbol{\eta}-\frac{1}{n}\left(\mathbf{u}^{n} \otimes \nabla_{x} \rho^{n}\right): \nabla_{x} \boldsymbol{\eta} \mathrm{d} x \mathrm{~d} t \\
& \quad+\int_{Q_{\tau}}\left(\left(1-H\left(\left|\mathbf{u}^{n}\right|^{2}\right)\right) \rho^{n} \mathbf{u}^{n} \cdot \boldsymbol{\eta}-\rho^{n} \int_{\Omega} \nabla_{x} K(x-y) \rho^{n}(y) \mathrm{d} y \cdot \boldsymbol{\eta}\right. \\
& \left.\quad+\rho^{n} \int_{\Omega} \psi(x-y) \rho^{n}(y)\left(\mathbf{u}^{n}(y)-\mathbf{u}^{n}(x)\right) \mathrm{d} y \cdot \boldsymbol{\eta}\right) \mathrm{d} x \mathrm{~d} t, \quad(2.2)
\end{aligned}
$$

for any test function $\boldsymbol{\eta} \in X_{n}$ and all $\tau \in[0, T]$, where $\rho^{n}=S_{\rho_{0}^{n}}\left(\mathbf{u}^{n}\right)$ is a unique solution to (2.1).

As the additional terms on the right-hand side of (2.2) can be treated in a standard way, we may directly use the method 4 of [8, Chapter 7] to claim the existence of a unique solution to (2.2) for any initial data that satisfy:

$$
\rho_{0}^{n} \in C^{2+\delta}(\Omega), \delta>0, \quad \inf _{\Omega} \rho_{0}^{n}>0 \quad \text { and } \quad \mathbf{m}_{0}^{n} \in L^{2}(\Omega) .
$$

\subsection{Limit in $n$}

The aim of this section is to tend with $n$ to infinity. In order to derive the energy estimates we use $\boldsymbol{\eta}=\mathbf{u}^{n}$ as a test function in (2.2) which together with (2.1) and the symmetry of $K$ and $\psi$ yield

$$
\begin{gathered}
\int_{\Omega}\left(\frac{1}{2} \rho^{n}\left|\mathbf{u}^{n}\right|^{2}+\right. \\
\left.+\int_{Q_{\tau}}\left(\varepsilon \mid \rho_{x}\right)+\left.\frac{1}{2} \rho^{n} K * \mathbf{u}^{n}\right|^{2}+\frac{1}{n} P^{\prime \prime}\left(\rho^{n}\right)\left|\nabla_{x} \rho^{n}\right|^{2}\right) \mathrm{d} x \mathrm{~d} t \\
\quad \leq \int_{\Omega}\left(\frac{1}{2} \frac{\left|\mathbf{m}_{0}^{n}\right|^{2}}{\rho_{0}^{n}}+P\left(\rho_{0}^{n}\right)+\frac{1}{2} \rho_{0}^{n} K * \rho_{0}^{n}\right) \mathrm{d} x \\
+\int_{Q_{\tau}}\left(1-H\left(\left|\mathbf{u}^{n}\right|^{2}\right)\right) \rho^{n}\left|\mathbf{u}^{n}\right|^{2} \mathrm{~d} x \mathrm{~d} t+\frac{1}{n} \int_{Q_{\tau}} \int_{\Omega} \rho^{n}(x) \Delta_{x} K(x-y) \rho^{n}(y) \mathrm{d} y \mathrm{~d} x \mathrm{~d} t \\
-\frac{1}{2} \int_{Q_{\tau}} \int_{\Omega} \rho^{n}(x) \rho^{n}(y) \psi(x-y)\left(\mathbf{u}^{n}(x)-\mathbf{u}^{n}(y)\right)^{2} \mathrm{~d} y \mathrm{~d} x \mathrm{~d} t, \quad(2.3)
\end{gathered}
$$

\footnotetext{
${ }^{4}$ The only difference is the energy inequality which can be replaced by 2.3 .
} 
for all $\tau \in[0, T]$. From now on, we shall consider $\rho_{0}^{n}$ and $\mathbf{m}_{0}^{n}$ such that $\rho_{0}^{n} \rightarrow \rho_{0}$ and $\mathbf{m}_{0}^{n} \rightarrow \rho_{0} \mathbf{u}_{0}$ in $L^{1}(\Omega)$ together with

$$
\int_{\Omega}\left(\frac{1}{2} \frac{\left|\mathbf{m}_{0}^{n}\right|^{2}}{\rho_{0}^{n}}+P\left(\rho_{0}^{n}\right)\right) \mathrm{d} x \rightarrow \int_{\Omega}\left(\frac{1}{2} \rho_{0}\left|\mathbf{u}_{0}\right|^{2}+P\left(\rho_{0}\right)\right) \mathrm{d} x .
$$

This possible due to (1.8) and [10, Section 7.10.7].

By integrating (2.1) over $\Omega$ we deduce that

$$
\sup _{t \in(0, T)}\left\|\rho^{n}(t)\right\|_{L^{1}(\Omega)}<c
$$

and since

$\frac{1}{n}\left|\int_{\Omega} \int_{\Omega} \rho^{n}(x) \Delta_{x} K(x-y) \rho^{n}(y) \mathrm{d} y \mathrm{~d} x\right| \leq \frac{1}{n}\left\|\Delta_{x} K\right\|_{L^{\infty}(\Omega)}\left\|\rho^{n}(t)\right\|_{L^{1}(\Omega)}^{2} \leq \frac{c}{n} \rightarrow 0$, we infer from (2.3) and Gronwall inequality that

$$
\begin{aligned}
\sup _{t \in(0, T)}\left\|\rho^{n}(t)\left|\mathbf{u}^{n}\right|^{2}(t)\right\|_{L^{1}(\Omega)} & \leq c, \\
\sup _{t \in(0, T)}\left\|P\left(\rho^{n}(t)\right)\right\|_{L^{1}(\Omega)} & \leq c, \\
\varepsilon\left\|\nabla_{x} \mathbf{u}^{n}\right\|_{L^{2}\left(Q_{T}\right)}^{2} & \leq c, \\
\frac{1}{n}\left\|P^{\prime \prime}\left(\rho^{n}\right)\left|\nabla_{x} \rho^{n}\right|^{2}\right\|_{L^{1}\left(Q_{T}\right)} & \leq c,
\end{aligned}
$$

with $c>0$ independent of $n$. Here and hereafter in this section, $c$ denotes a positive generic constant that is independent of $\varepsilon$ and $n$ unless specified otherwise.

From (1.2) and (2.5) 4 we derive

$$
\begin{array}{r}
\sup _{t \in(0, T)} \int_{\Omega} \rho^{n} \log \rho^{n} \mathrm{~d} x \leq c, \\
\frac{1}{n} \int_{0}^{T} \int_{\left\{\rho^{n}>1\right\}} \frac{\left|\nabla_{x} \rho^{n}\right|^{2}}{\rho^{n}} \mathrm{~d} x \mathrm{~d} t \leq c .
\end{array}
$$

As $\rho^{n}$ is smooth enough, we may multiply (2.1) by $b^{\prime}\left(\rho^{n}\right)$ for any $b \in C_{c}^{\infty}([0, \infty))$ in order to get

$$
\begin{aligned}
\partial_{t} b\left(\rho^{n}\right)+\operatorname{div}_{x}\left(b\left(\rho^{n}\right) \mathbf{u}^{n}\right)+ & \left(b^{\prime}\left(\rho^{n}\right) \rho^{n}-b\left(\rho^{n}\right)\right) \operatorname{div}_{x} \mathbf{u}^{n} \\
& =\frac{1}{n} \operatorname{div}_{x}\left(b^{\prime}\left(\rho^{n}\right) \nabla_{x} \rho^{n}\right)-\frac{1}{n} b^{\prime \prime}\left(\rho^{n}\right)\left|\nabla_{x} \rho^{n}\right|^{2} \text { in } Q_{T} .
\end{aligned}
$$


By choosing a suitable $b$, namely $b(z)=z^{2}$ for $z \leq 1$, and integrating over $Q_{T}$ we get

$$
\frac{1}{n} \int_{0}^{T} \int_{\left\{\rho^{n} \leq 1\right\}}\left|\nabla_{x} \rho^{n}\right|^{2} \mathrm{~d} x \mathrm{~d} t \leq c(\varepsilon) .
$$

Next we obtain relative compactness in $L^{1}$. First, we deduce from (2.6) 1 that $\left\{\rho^{n}\right\}$ is equi-integrable. Indeed, we have

$$
\log k \int_{\left\{\rho^{n} \geq k\right\}} \rho^{n} \mathrm{~d} x \leq \int_{\left\{\rho^{n} \geq k\right\}} \rho^{n} \log \rho^{n} \mathrm{~d} x \leq c, \quad \forall k \in(0, \infty),
$$

yielding that $\sup _{n} \int_{\left\{\rho^{n} \geq k\right\}} \rho^{n} \mathrm{~d} x \rightarrow 0$ as $k \rightarrow \infty$. Second, the sequence $\left\{\rho^{n} \mathbf{u}^{n}\right\}$ is also equi-integrable. To see this it is enough to consider the following sequence of inequalities, which holds for every $k, m \in(0, \infty)$,

$$
\begin{aligned}
& \int_{\left\{\left|\rho^{n} \mathbf{u}^{n}\right| \geq k\right\}}\left|\rho^{n} \mathbf{u}^{n}\right| \mathrm{d} x \leq \int_{\left\{\rho^{n} \geq \frac{k}{m}\right\}}\left|\rho^{n} \mathbf{u}^{n}\right| \mathrm{d} x+\int_{\left\{\left|\mathbf{u}^{n}\right| \geq m\right\}}\left|\rho^{n} \mathbf{u}^{n}\right| \mathrm{d} x \\
\leq & \frac{1}{\sqrt{\log \frac{k}{m}}} \int_{\Omega} \sqrt{\rho^{n} \log \rho^{n}} \sqrt{\rho}^{n} \mathbf{u}^{n} \mathrm{~d} x+\frac{1}{m} \int_{\Omega} \rho^{n}\left|\mathbf{u}^{n}\right|^{2} \mathrm{~d} x \leq c\left(\frac{1}{\sqrt{\log \frac{k}{m}}}+\frac{1}{m}\right) .
\end{aligned}
$$

As the previous estimate holds for every choice of $k$ and $m$, it suffices to consider $m=\sqrt{k}$ in order to deduce

$$
\sup _{n} \int_{\left\{\left|\rho^{n} \mathbf{u}^{n}\right|>k\right\}}\left|\rho^{n} \mathbf{u}^{n}\right| \mathrm{d} x \rightarrow 0
$$

as $k \rightarrow \infty$. Third, we can claim the equi-integrability of $\left\{\mathbf{u}^{n}\right\}$ using the Poincaré inequality which can be found, e.g., in [8, Lemma 3.2].

Now we are able to proceed to limit in a weak formulation of the continuity equation (2.1), i.e., in

$$
\begin{array}{r}
\int_{\Omega} \rho^{n}(\tau) \varphi(\tau) \mathrm{d} x-\int_{\Omega} \rho_{0}^{n} \varphi(0) \mathrm{d} x-\int_{Q_{\tau}} \rho^{n} \partial_{t} \varphi \mathrm{d} x \mathrm{~d} t-\int_{Q_{\tau}} \rho^{n} \mathbf{u}^{n} \nabla_{x} \varphi \mathrm{d} x \mathrm{~d} t \\
=-\frac{1}{n} \int_{Q_{\tau}} \nabla_{x} \rho^{n} \nabla_{x} \varphi \mathrm{d} x \mathrm{~d} t,
\end{array}
$$

which holds for every $\varphi \in C^{1}([0, T], \Omega)$ and $\tau \in[0, T]$. By (2.4), (2.6) 2 and (2.7) 
the right-hand side of (2.8) vanishes as $n \rightarrow \infty$ since

$$
\begin{aligned}
\mid \frac{1}{n} \int_{0}^{T} \int_{\left\{\rho^{n}>1\right\}} & \frac{1}{\sqrt{\rho^{n}}} \nabla_{x} \rho^{n} \sqrt{\rho^{n}} \nabla_{x} \varphi \mathrm{d} x \mathrm{~d} t+\frac{1}{n} \int_{0}^{T} \int_{\left\{\rho^{n} \leq 1\right\}} \nabla_{x} \rho^{n} \nabla_{x} \varphi \mathrm{d} x \mathrm{~d} t \mid \\
\leq & \frac{1}{\sqrt{n}}\left(\frac{1}{n} \int_{0}^{T} \int_{\left\{\rho^{n}>1\right\}} \frac{\left|\nabla_{x} \rho^{n}\right|^{2}}{\rho^{n}} \mathrm{~d} x \mathrm{~d} t\right)^{\frac{1}{2}}\left\|\nabla_{x} \varphi\right\|_{L^{\infty}(\Omega)}\left\|\rho^{n}\right\|_{L^{1}\left(Q_{T}\right)}^{\frac{1}{2}} \\
& +\frac{1}{\sqrt{n}}\left(\frac{1}{n} \int_{0}^{T} \int_{\left\{\rho^{n} \leq 1\right\}}\left|\nabla_{x} \rho^{n}\right|^{2} \mathrm{~d} x \mathrm{~d} t\right)^{\frac{1}{2}}\left\|\nabla_{x} \varphi\right\|_{L^{2}\left(Q_{T}\right)} \leq \frac{1}{\sqrt{n}} c(\varepsilon) .
\end{aligned}
$$

Next we may use [11, Theorem 6.2] and there exists a Young measure

$$
\nu_{t, x} \in \mathcal{P}\left([0, \infty) \times \mathbb{R}^{N}\right) \text { for a.a. }(t, x) \in[0, T] \times \Omega,
$$

associated with the equi-integrable sequence $\left\{\rho^{n}, \mathbf{u}^{n}\right\}$. We will use the notation $\rho(t, x)=\left\langle\nu_{t, x}, s\right\rangle$ and $\mathbf{u}(t, x)=\left\langle\nu_{t, x}, \mathbf{v}\right\rangle$ for shortness. As we proceed to limit in (2.8) we get

$$
\int_{\Omega} \rho(\tau) \varphi(\tau) \mathrm{d} x-\int_{Q_{\tau}} \rho \partial_{t} \varphi \mathrm{d} x \mathrm{~d} t-\int_{Q_{\tau}}\left\langle\nu_{t, x}, s \mathbf{v}\right\rangle \nabla_{x} \varphi(t, x) \mathrm{d} x \mathrm{~d} t=\int_{\Omega} \rho_{0} \varphi(0) \mathrm{d} x,
$$

which holds for every $\varphi \in C^{1}([0, T], \Omega)$ and all $\tau \in[0, T]$ as $\rho^{n}$ is precompact in $C_{\text {weak }}\left([0, T], L^{1}(\Omega)\right)$.

Next, we show that we can proceed to limit in the balance of energy (2.3). Due to $L^{1}$-bounds from (2.5) we get

$$
\begin{aligned}
\frac{1}{2} \rho^{n}\left|\mathbf{u}^{n}\right|^{2}+P\left(\rho^{n}\right) & \rightarrow\left\langle\nu_{t, x}, \frac{1}{2} s|\mathbf{v}|^{2}+P(s)\right\rangle+\xi \text { weakly* in } L_{\text {weak }}^{\infty}(0, T, \mathcal{M}(\Omega)), \\
\left|\nabla_{x} \mathbf{u}^{n}\right|^{2} & \rightarrow\left|\nabla_{x} \mathbf{u}\right|^{2}+\sigma \text { weakly }^{*} \text { in } \mathcal{M}^{+}([0, T] \times \Omega),
\end{aligned}
$$

for some non-negative measures $\xi$ and $\sigma$. Further, since $K$ is smooth and $\left\{\rho^{n}\right\}$ is equi-integrable we obtain

$$
K * \rho^{n} \rightarrow K * \rho \text { a.e. in } Q_{T} \text { and }\left\|K * \rho^{n}\right\|_{L^{\infty}\left(Q_{T}\right)} \leq c .
$$

We use Young inequality with the functions $\Phi(t)=(t+1) \log (t+1)-t$ and $\Psi(t)=e^{t}-t-1$ in order to derive

$$
\int_{\Omega} \rho^{n}\left(K * \rho^{n}-K * \rho\right) \mathrm{d} x \leq \delta \int_{\Omega} \Phi\left(\rho^{n}\right) \mathrm{d} x+c(\delta) \int_{\Omega} \Psi\left(K * \rho^{n}-K * \rho\right) \mathrm{d} x,
$$


for arbitrary $\delta>0$. Since the second term on the right-hand side tends to zero as $n \rightarrow \infty$ due to Lebesgue's dominated convergence theorem and the first term can be estimated by $\delta c$, we immediately conclude that

$$
\frac{1}{2} \int_{\Omega}\left(\rho^{n} K * \rho^{n}\right)(\tau) \mathrm{d} x \rightarrow \frac{1}{2} \int_{\Omega}(\rho K * \rho)(\tau) \mathrm{d} x,
$$

for all $\tau \in[0, T]$. Since $P^{\prime \prime}(z)=\frac{p^{\prime}(z)}{z}>0$ we neglect the term $\frac{1}{n} \int_{Q_{\tau}} P^{\prime \prime}\left(\rho^{n}\right)\left|\nabla_{x} \rho^{n}\right|^{2} \mathrm{~d} x \mathrm{~d} t$.

Next, we get

$\int_{Q_{\tau}}\left(1-H\left(\left|\mathbf{u}^{n}\right|^{2}\right)\right) \rho^{n}\left|\mathbf{u}^{n}\right|^{2} \mathrm{~d} x \mathrm{~d} t \rightarrow \int_{Q_{\tau}}\left\langle\nu_{t, x},\left(1-H\left(|\mathbf{v}|^{2}\right)\right) s|\mathbf{v}|^{2}\right\rangle \mathrm{d} x \mathrm{~d} t+\int_{0}^{\tau} \kappa(t)|\Omega| \mathrm{d} t$,

for all $\tau \in[0, T]$ and some measure $\kappa$. Due to [7, Lemma 2.1] and boundedness

of $H$ we have $|\kappa(t)| \leq c \xi(t)$ for all $t \in[0, T]$.

In order to derive the convergence in the last term of (2.3) it is enough to realize that the Arzela-Ascoli theorem yields for all $t \in[0, T]$ that

$$
\begin{aligned}
\psi * \rho^{n} & \rightrightarrows \psi * \rho \text { on } \Omega \\
\psi *\left(\rho^{n} \mathbf{u}^{n}\right) & \rightrightarrows \psi *\left\langle\nu_{t, y}, s \mathbf{v}\right\rangle \text { on } \Omega
\end{aligned}
$$

as far as $\psi \in C^{1}(\Omega)$. Thus we may infer that

$$
\begin{aligned}
& \lim _{n \rightarrow \infty}\left(\int_{\Omega} \int_{\Omega} \rho^{n}(x) \rho^{n}(y) \psi(x-y)\left|\mathbf{u}^{n}(x)\right|^{2} \mathrm{~d} y \mathrm{~d} x\right. \\
& \left.\left.-\int_{\Omega} \int_{\Omega} \rho^{n}(x) \rho^{n}(y) \psi(x-y) \mathbf{u}^{n}(x) \mathbf{u}^{n}(y)\right) \mathrm{d} y \mathrm{~d} x\right)(t) \\
& \quad=\int_{\Omega} \int_{\Omega}\left\langle\nu_{t, x}, s|\mathbf{v}|^{2}\right\rangle \rho(t, y) \psi(x-y) \mathrm{d} y \mathrm{~d} x \\
& \quad-\int_{\Omega} \int_{\Omega}\left\langle\nu_{t, x}, s \mathbf{v}\right\rangle \psi(x-y)\left\langle\nu_{t, y}, s \mathbf{v}\right\rangle \mathrm{d} y \mathrm{~d} x+\int_{\Omega} \psi * \rho(t) \mathrm{d} \lambda(t),
\end{aligned}
$$

where $\lambda$ is a non-negative measure satisfying $\lambda(t) \leq c \xi(t)$ for all $t \in[0, T]$. 
As a consequence of the previous ideas we get that (2.3) converges to

$$
\begin{gathered}
\int_{\Omega}\left\langle\nu_{\tau, x}, \frac{1}{2} s|\mathbf{v}|^{2}+P(s)\right\rangle \mathrm{d} x+\frac{1}{2} \int_{\Omega}(\rho K * \rho)(\tau) \mathrm{d} x \\
+\varepsilon \int_{Q_{\tau}}\left|\nabla_{x} \mathbf{u}\right|^{2} \mathrm{~d} x \mathrm{~d} t+\xi(\tau)|\Omega| \\
\leq \int_{\Omega}\left(\frac{1}{2} \rho_{0}\left|\mathbf{u}_{0}\right|^{2}+P\left(\rho_{0}\right)+\frac{1}{2} \rho_{0}\left(K * \rho_{0}\right)\right) \mathrm{d} x \\
+\int_{Q_{\tau}}\left\langle\nu_{t, x},\left(1-H\left(|\mathbf{v}|^{2}\right)\right) s|\mathbf{v}|^{2}\right\rangle \mathrm{d} x \mathrm{~d} t+c \int_{0}^{\tau} \xi(t)|\Omega| \mathrm{d} t \\
-\int_{Q_{\tau}} \int_{\Omega}\left(\left\langle\nu_{t, x}, s|\mathbf{v}|^{2}\right\rangle \rho(t, y) \psi(x-y)-\left\langle\nu_{t, x}, s \mathbf{v}\right\rangle \psi(x-y)\left\langle\nu_{t, y}, s \mathbf{v}\right\rangle\right) \mathrm{d} y \mathrm{~d} x \mathrm{~d} t
\end{gathered}
$$

for a.a. $\tau \in(0, T)$.

It remains to proceed to limit in the momentum equation (2.2). Nevertheless, the term $\frac{1}{n} \int_{Q_{\tau}}\left(\mathbf{u}^{n} \otimes \nabla_{x} \rho^{n}\right): \nabla_{x} \boldsymbol{\varphi} \mathrm{d} x \mathrm{~d} t$ vanishes due to $L^{2}$ estimates following from $(2.5)_{1},(2.6)_{2}, 2.7$ and Poincaré inequality and all the other terms are equi-integrable except of $\int_{Q_{\tau}} p\left(\rho^{n}\right) \operatorname{div}_{x} \boldsymbol{\varphi} \mathrm{d} x \mathrm{~d} t$ and $\int_{Q_{\tau}}\left(\rho^{n} \mathbf{u}^{n} \otimes \mathbf{u}^{n}\right): \nabla_{x} \boldsymbol{\varphi} \mathrm{d} x \mathrm{~d} t$. However, since $\left|\rho^{n} \mathbf{u}^{n} \otimes \mathbf{u}^{n}\right| \leq \rho^{n}\left|\mathbf{u}^{n}\right|^{2}$ and $p\left(\rho^{n}\right) \leq a P\left(\rho^{n}\right)$ for $\rho^{n}$ sufficiently large and some $a>0$, we get

$$
\begin{gathered}
\int_{\Omega}\left\langle\nu_{\tau, x}, s \mathbf{v}\right\rangle \boldsymbol{\varphi}(\tau, x) \mathrm{d} x-\int_{Q_{\tau}}\left\langle\nu_{t, x}, s \mathbf{v}\right\rangle \partial_{t} \boldsymbol{\varphi}(t, x) \mathrm{d} x \mathrm{~d} t \\
-\int_{Q_{\tau}}\left\langle\nu_{t, x}, s \mathbf{v} \otimes \mathbf{v}\right\rangle \nabla_{x} \boldsymbol{\varphi}(t, x) \mathrm{d} x \mathrm{~d} t+\varepsilon \int_{Q_{\tau}} \nabla_{x} \mathbf{u}: \nabla_{x} \varphi \mathrm{d} x \mathrm{~d} t \\
-\int_{Q_{\tau}}\left\langle\nu_{t, x}, p(s)\right\rangle \operatorname{div}_{x} \boldsymbol{\varphi}(x) \mathrm{d} x \mathrm{~d} t=\int_{Q_{\tau}}\left\langle\nu_{t, x},\left(1-H\left(|\mathbf{v}|^{2}\right)\right) s \mathbf{v}\right\rangle \boldsymbol{\varphi}(t, x) \mathrm{d} x \mathrm{~d} t \\
-\int_{Q_{\tau}} \rho(t, x) \int_{\Omega} \nabla_{x} K(x-y) \rho(t, y) \mathrm{d} y \boldsymbol{\varphi}(t, x) \mathrm{d} x \mathrm{~d} t \\
+\int_{Q_{\tau}} \rho(t, x) \int_{\Omega} \psi(x-y)\left\langle\nu_{t, y}, s \mathbf{v}\right\rangle \mathrm{d} y \boldsymbol{\varphi}(t, x) \mathrm{d} x \mathrm{~d} t \\
-\int_{Q_{\tau}}\left\langle\nu_{t, x}, s \mathbf{v}\right\rangle \int_{\Omega} \psi(x-y) \rho(t, y) \mathrm{d} y \boldsymbol{\varphi}(t, x) \mathrm{d} x \mathrm{~d} t \\
+\int_{\Omega} \rho_{0} \mathbf{u}_{0} \boldsymbol{\varphi}(0) \mathrm{d} x+\int_{0}^{\tau} \int_{\Omega} \nabla_{x} \boldsymbol{\varphi}(t) \mathrm{d} \mu(t) \mathrm{d} t
\end{gathered}
$$

where, due to [7, Lemma 2.1], $\mu$ is a measure such that $|\mu(t)| \leq c \xi(t)$ for all $t \in[0, T]$.

This concludes the proof of Theorem 1.3 . 


\section{Relative entropy inequality}

Let $\left(\nu^{\varepsilon}, \mathcal{D}^{\varepsilon}\right)$ be a measure-valued solution to (1.6) and (1.7). Similarly as in [7, Section 3] one can show that every pair of smooth functions $(r, \mathbf{U}) \in$ $C^{1}([0, T] \times \Omega) \times C^{1}([0, T] \times \Omega)^{N}$ satisfies

$$
\begin{aligned}
& \mathcal{E}\left(\nu_{\tau, \cdot}^{\varepsilon}, r(\tau), \mathbf{U}(\tau)\right)+\mathcal{D}_{\varepsilon}(\tau)+\int_{\Omega} \frac{1}{2}\left(\left(\rho^{\varepsilon}-r\right) K *\left(\rho^{\varepsilon}-r\right)\right)(\tau) \mathrm{d} x \\
& +\varepsilon \int_{Q_{\tau}} \nabla_{x}\left(\mathbf{u}^{\varepsilon}-\mathbf{U}\right): \nabla_{x}\left(\mathbf{u}^{\varepsilon}-\mathbf{U}\right) \mathrm{d} x \mathrm{~d} t \leq \mathcal{E}\left(\nu_{0}^{\varepsilon}, r(0), \mathbf{U}(0)\right) \\
& \quad+\int_{\Omega} \frac{1}{2}\left(\left(\rho^{\varepsilon}-r\right) K *\left(\rho^{\varepsilon}-r\right)\right)(0) \mathrm{d} x+\mathcal{R}\left(\nu_{\tau, \cdot}^{\varepsilon}, r(\tau), \mathbf{U}(\tau)\right),
\end{aligned}
$$

where

$$
\begin{gathered}
\mathcal{R}\left(\nu_{\tau, \cdot}^{\varepsilon}, r(\tau), \mathbf{U}(\tau)\right)=\int_{Q_{\tau}}\left(\left\langle\nu_{t, x}^{\varepsilon}, s(\mathbf{U}-\mathbf{v})\right\rangle \partial_{t} \mathbf{U}+\left\langle\nu_{t, x}^{\varepsilon}, s \mathbf{v} \otimes(\mathbf{U}-\mathbf{v})\right\rangle \nabla_{x} \mathbf{U}\right) \mathrm{d} x \mathrm{~d} t \\
\quad+\varepsilon \int_{Q_{\tau}} \nabla_{x} \mathbf{U}: \nabla_{x}\left(\mathbf{U}-\mathbf{u}^{\varepsilon}\right) \mathrm{d} x \mathrm{~d} t \\
+\int_{Q_{\tau}}\left(\left(1-\frac{\rho^{\varepsilon}}{r}\right) p^{\prime}(r) \partial_{t} r-\left\langle\nu_{t, x}^{\varepsilon}, s \mathbf{v}\right\rangle \frac{p^{\prime}(r)}{r} \nabla_{x} r+\left\langle\nu_{t, x}^{\varepsilon}, p(s)\right\rangle \operatorname{div}_{x} \mathbf{U}\right) \mathrm{d} x \mathrm{~d} t \\
+\int_{Q_{\tau}}\left\langle\nu_{t, x}^{\varepsilon}, s \mathbf{v}(\mathbf{v}-\mathbf{U})\left(1-H\left(|\mathbf{v}|^{2}\right)\right)\right\rangle \mathrm{d} x \mathrm{~d} t+\int_{Q_{\tau}}\left(\rho^{\varepsilon} \nabla_{x} K * \rho^{\varepsilon}\right) \cdot \mathbf{U} \mathrm{d} x \mathrm{~d} t \\
-\frac{1}{2} \int_{\Omega}\left(\left(r K * \rho^{\varepsilon}+\left(\rho^{\varepsilon}-r\right) K * r\right)(\tau)-\left(r K * \rho^{\varepsilon}+\left(\rho^{\varepsilon}-r\right) K * r\right)(0)\right) \mathrm{d} x \\
+\int_{Q_{\tau}} \int_{\Omega}\left\langle\nu_{t, x}^{\varepsilon}, s(\mathbf{v}-\mathbf{U}(t, x))\right\rangle\left\langle\nu_{t, y}^{\varepsilon}, s \mathbf{v}\right\rangle \psi(x-y) \mathrm{d} y \mathrm{~d} x \mathrm{~d} t \\
-\int_{Q_{\tau}} \int_{\Omega}\left\langle\nu_{t, x}^{\varepsilon}, s \mathbf{v}(\mathbf{v}-\mathbf{U}(t, x))\right\rangle \rho^{\varepsilon}(t, y) \psi(x-y) \mathrm{d} y \mathrm{~d} x \mathrm{~d} t \\
+\int_{0}^{\tau}\left(\int_{\Omega} \nabla_{x} \mathbf{U} \mathrm{d} \mu^{\varepsilon}(t)+c \mathcal{D}^{\varepsilon}(t)\right) \mathrm{d} t
\end{gathered}
$$

for a.a. $\tau \in(0, T)$ and $c>0$ independent of $\varepsilon$ and any solution. 


\section{Inviscid limit}

Let $(r, \mathbf{U})$ be a strong solution to (1.1) and (1.5). Consequently,

$$
\begin{aligned}
& \int_{Q_{\tau}}\left(\rho^{\varepsilon} \nabla_{x} K * \rho^{\varepsilon}\right) \cdot \mathbf{U} \mathrm{d} x \mathrm{~d} t \\
&=\int_{Q_{\tau}}\left(\left(\rho^{\varepsilon}-r\right) \nabla_{x} K *\left(\rho^{\varepsilon}-r\right) \cdot \mathbf{U}+r \nabla_{x} K *\left(\rho^{\varepsilon}-r\right) \cdot \mathbf{U}+\left(\rho^{\varepsilon} \nabla_{x} K * r\right) \cdot \mathbf{U}\right) \mathrm{d} x \mathrm{~d} t \\
&=\int_{Q_{\tau}}\left(\left(\rho^{\varepsilon}-r\right) \mathbf{U} \cdot \nabla_{x} K *\left(\rho^{\varepsilon}-r\right)+\partial_{t} r K *\left(\rho^{\varepsilon}-r\right)\right. \\
&\left.\quad+\left\langle\nu_{t, x}^{\varepsilon}, s(\mathbf{U}-\mathbf{v})\right\rangle \nabla_{x} K * r+\partial_{t} \rho^{\varepsilon} K * r\right) \mathrm{d} x \mathrm{~d} t .
\end{aligned}
$$

We rewrite (3.1) and (3.2) in the following way

$$
\begin{aligned}
& \mathcal{E}\left(\nu_{\tau, \cdot}^{\varepsilon}, r(\tau), \mathbf{U}(\tau)\right)+\varepsilon \int_{Q_{\tau}}\left|\nabla_{x}\left(\mathbf{u}^{\varepsilon}-\mathbf{U}\right)\right|^{2} \mathrm{~d} x \mathrm{~d} t+\mathcal{D}^{\varepsilon}(\tau) \\
& +\frac{1}{2} \int_{\Omega}\left(\left(\left(\rho^{\varepsilon}-r\right) K *\left(\rho^{\varepsilon}-r\right)\right)(\tau)-\left(\left(\rho^{\varepsilon}-r\right) K *\left(\rho^{\varepsilon}-r\right)\right)(0)\right) \mathrm{d} x \\
& \leq \mathcal{E}\left(\nu_{0}^{\varepsilon}, r_{0}, \mathbf{U}_{0}\right) \\
& +\int_{Q_{\tau}}\left(\left\langle\nu_{t, x}^{\varepsilon}, s(\mathbf{U}-\mathbf{v})\right\rangle \partial_{t} \mathbf{U}+\left\langle\nu_{t, x}^{\varepsilon}, s \mathbf{v} \otimes(\mathbf{U}-\mathbf{v})\right\rangle \nabla_{x} \mathbf{U}\right) \mathrm{d} x \mathrm{~d} t \\
& +\varepsilon \int_{Q_{\tau}} \nabla_{x} \mathbf{U}: \nabla_{x}\left(\mathbf{U}-\mathbf{u}^{\varepsilon}\right) \mathrm{d} x \mathrm{~d} t \\
& +\int_{Q_{\tau}}\left\langle\nu_{t, x}^{\varepsilon}, s \mathbf{U}-s \mathbf{v}\right\rangle \frac{p^{\prime}(r)}{r} \nabla_{x} r \mathrm{~d} x \mathrm{~d} t \\
& -\int_{Q_{\tau}}\left\langle\nu_{t, x}^{\varepsilon}, p(s)-p^{\prime}(r)(s-r)-p(r)\right\rangle \operatorname{div}_{x} \mathbf{U} \mathrm{d} x \mathrm{~d} t \\
& +\int_{Q_{\tau}}\left\langle\nu_{t, x}^{\varepsilon}, s \mathbf{v}(\mathbf{v}-\mathbf{U})\left(1-H\left(|\mathbf{v}|^{2}\right)\right)\right\rangle \mathrm{d} x \mathrm{~d} t \\
& +\int_{Q_{\tau}}\left(\left(\rho^{\varepsilon}-r\right) \mathbf{U} \nabla_{x} K *\left(\rho^{\varepsilon}-r\right)+\left\langle\nu_{t, x}^{\varepsilon}, s(\mathbf{U}-\mathbf{v})\right\rangle \nabla_{x} K * r\right) \mathrm{d} x \mathrm{~d} t \\
& +\int_{Q_{\tau}} \int_{\Omega}\left\langle\nu_{t, x}^{\varepsilon}, s(\mathbf{v}-\mathbf{U}(t, x))\right\rangle\left\langle\nu_{t, y}^{\varepsilon}, s \mathbf{v}\right\rangle \psi(x-y) \mathrm{d} y \mathrm{~d} x \mathrm{~d} t \\
& -\int_{Q_{\tau}} \int_{\Omega}\left\langle\nu_{t, x}^{\varepsilon}, s \mathbf{v}(\mathbf{v}-\mathbf{U}(t, x))\right\rangle \rho^{\varepsilon}(t, y) \psi(x-y) \mathrm{d} y \mathrm{~d} x \mathrm{~d} t \\
& +\int_{0}^{\tau}\left(\int_{\Omega} \nabla_{x} \mathbf{U} \mathrm{d} \mu^{\varepsilon}(t)+c \mathcal{D}^{\varepsilon}(t)\right) \mathrm{d} t=: \sum_{i=1}^{10} \mathcal{I}_{i},
\end{aligned}
$$

for a.a. $\tau \in(0, T)$. Here and hereafter $c$ denotes a positive generic constant independent of $\varepsilon$. 


\subsection{Estimates of $\mathcal{I}_{i}$}

Since we assume that $\mathcal{E}\left(\nu_{0}^{\varepsilon}, r_{0}, \mathbf{U}_{0}\right) \rightarrow 0$ as $\varepsilon \rightarrow 0$, we get

$$
\mathcal{I}_{1} \leq \Gamma(\varepsilon)
$$

where $\Gamma(\varepsilon) \rightarrow 0$ as $\varepsilon \rightarrow 0$.

Further, as $\mathbf{U}$ is smooth, we deduce

$$
\mathcal{I}_{3} \leq \varepsilon \frac{c}{2}+\varepsilon \frac{1}{2} \int_{Q_{\tau}}\left|\nabla_{x}\left(\mathbf{u}_{\varepsilon}-\mathbf{U}\right)\right|^{2} \mathrm{~d} x \mathrm{~d} t
$$

According to estimates on $\mu^{\varepsilon}$ and smoothness of $\mathbf{U}$, we get

$$
\mathcal{I}_{10} \leq c \int_{0}^{\tau} \mathcal{D}^{\varepsilon}(t) \mathrm{d} t
$$

Due to (1.1) 1

$$
\begin{gathered}
\mathcal{I}_{2}+\mathcal{I}_{4}+\mathcal{I}_{7}=\int_{Q_{\tau}}\left[\left\langle\nu_{t, x}^{\varepsilon}, s(\mathbf{U}-\mathbf{v})\right\rangle \cdot\right. \\
\cdot\left(-\mathbf{U} \nabla_{x} \mathbf{U}+\left(1-H\left(|\mathbf{U}|^{2}\right)\right) \mathbf{U}+\int_{\Omega} \psi(x-y) r(y)(\mathbf{U}(y)-\mathbf{U}(x)) \mathrm{d} y\right) \\
\left.+\left\langle\nu_{t, x}^{\varepsilon}, s \mathbf{v} \otimes(\mathbf{U}-\mathbf{v})\right\rangle \nabla_{x} \mathbf{U}+\left(\rho^{\varepsilon}-r\right) \mathbf{U} \nabla_{x} K *\left(\rho^{\varepsilon}-r\right)\right] \mathrm{d} x \mathrm{~d} t \\
=\int_{Q_{\tau}}\left\langle\nu_{t, x}^{\varepsilon}, s(\mathbf{v}-\mathbf{U}) \nabla_{x} \mathbf{U}(\mathbf{U}-\mathbf{v})\right\rangle \mathrm{d} x \mathrm{~d} t \\
+\int_{Q_{\tau}}\left\langle\nu_{t, x}^{\varepsilon}, s(\mathbf{U}-\mathbf{v})\right\rangle\left(1-H\left(|\mathbf{U}|^{2}\right)\right) \mathbf{U} \mathrm{d} x \mathrm{~d} t \\
+\int_{Q_{\tau}}\left\langle\nu_{t, x}^{\varepsilon}, s(\mathbf{U}-\mathbf{v})\right\rangle \int_{\Omega} \psi(x-y) r(y)(\mathbf{U}(y)-\mathbf{U}(x)) \mathrm{d} y \mathrm{~d} x \mathrm{~d} t \\
\quad+\int_{Q_{\tau}}\left(\rho^{\varepsilon}-r\right) \mathbf{U} \nabla_{x} K *\left(\rho^{\varepsilon}-r\right) \mathrm{d} x \mathrm{~d} t \\
=: \mathcal{I}_{11}+\mathcal{I}_{12}+\mathcal{I}_{13}+\mathcal{I}_{14} .
\end{gathered}
$$

The Taylor formula and [9, (4.1)] yield

$$
\left|p(s)-p^{\prime}(r)(s-r)-p(r)\right| \leq c\left|P(s)-P^{\prime}(r)(s-r)-P(r)\right|,
$$

which together with the definition of $\mathcal{E}$ imply

$$
\mathcal{I}_{5}+\mathcal{I}_{11} \leq c \int_{0}^{\tau} \mathcal{E}\left(\nu_{t, \cdot}^{\varepsilon}, r(t), \mathbf{U}(t)\right) \mathrm{d} t
$$


Further,

$$
\mathcal{I}_{6}+\mathcal{I}_{12}=\int_{Q_{\tau}}\left\langle\nu_{t, x}^{\varepsilon}, s(\mathbf{v}-\mathbf{U})\left(\mathbf{v}\left(1-H\left(|\mathbf{v}|^{2}\right)\right)-\mathbf{U}\left(1-H\left(|\mathbf{U}|^{2}\right)\right)\right)\right\rangle \mathrm{d} x \mathrm{~d} t .
$$

Due to the assumptions on $H$ it is possible to split it into two parts, namely $H=H_{1}+H_{2}$, where $H_{1} \in C^{1}([0, \infty))$ has a compact support and $H_{2}$ is nondecreasing. It then follows that

$$
-s(\mathbf{v}-\mathbf{U})\left(v H_{2}\left(|\mathbf{v}|^{2}\right)-\mathbf{U} H_{2}\left(|\mathbf{U}|^{2}\right)\right) \leq 0 .
$$

Since $H_{1}$ is a Lipschitz function with compact support, we get

$$
\left|H_{1}\left(|\mathbf{v}|^{2}\right)-H_{1}\left(|\mathbf{U}|^{2}\right)\right| \leq c|\mathbf{v}-\mathbf{U}|
$$

and thus

$$
\begin{aligned}
\left\langle\nu_{t, x}^{\varepsilon}, s(\mathbf{v}-\mathbf{U})\left(\mathbf{v} H_{1}\left(|\mathbf{v}|^{2}\right)-\right.\right. & \left.\left.\mathbf{U} H_{1}\left(|\mathbf{U}|^{2}\right)\right)\right\rangle \\
& \leq\left\langle\nu_{t, x}^{\varepsilon} s(\mathbf{v}-\mathbf{U})^{2} H_{1}\left(|\mathbf{v}|^{2}\right)\right\rangle+c\left\langle\nu_{t, x}^{\varepsilon}, s(\mathbf{v}-\mathbf{U})^{2}\right\rangle .
\end{aligned}
$$

Consequently,

$$
\mathcal{I}_{6}+\mathcal{I}_{12} \leq \int_{0}^{\tau} \mathcal{E}\left(\nu_{t, \cdot}^{\varepsilon}, r(t), \mathbf{U}(t)\right) \mathrm{d} t
$$

Further,

$$
\begin{gathered}
\mathcal{I}_{8}+\mathcal{I}_{9}+\mathcal{I}_{13}=\int_{Q_{\tau}} \int_{\Omega} \psi(x-y)\left\langle\nu_{t, x}^{\varepsilon}, s(\mathbf{v}-\mathbf{U})\right\rangle\left\langle\nu_{t, y}^{\varepsilon},(s-r)(\mathbf{U}-\mathbf{U}(x))\right\rangle \mathrm{d} y \mathrm{~d} x \mathrm{~d} t \\
-\frac{1}{2} \int_{Q_{\tau}} \int_{\Omega} \psi(x-y)\left(\left\langle\nu_{t, x}^{\varepsilon}, s(\mathbf{v}-\mathbf{U})^{2}\right\rangle \rho^{\varepsilon}(y)-\left\langle\nu_{t, x}^{\varepsilon}, s(\mathbf{v}-\mathbf{U})\right\rangle\left\langle\nu_{t, y}^{\varepsilon}, s(\mathbf{v}-\mathbf{U})\right\rangle\right) \mathrm{d} y \mathrm{~d} x \mathrm{~d} t .
\end{gathered}
$$

The second term on the right-hand side is negative by the same argument as in Remark 1.2, By the Young's inequality we deduce that

$$
\left|\int_{(0, \infty) \times \mathbb{R}^{N}} s(\mathbf{v}-\mathbf{U}) f(t, x, y) d \nu_{t, x}^{\varepsilon}\right| \leq \frac{1}{2} \int_{(0, \infty) \times \mathbb{R}^{N}} s(\mathbf{v}-\mathbf{U})^{2} d \nu_{t, x}^{\varepsilon}+\frac{1}{2} \rho^{\varepsilon} f^{2}(t, x, y),
$$

for any $f$ independent of $(s, \mathbf{v})$. This together with (1.10) implies

$$
\begin{aligned}
\mathcal{I}_{8}+\mathcal{I}_{9}+\mathcal{I}_{13} \leq \frac{1}{2} \int_{Q_{\tau}}\left\langle\nu_{t, x}^{\varepsilon}, s(\mathbf{v}-\mathbf{U})^{2}\right\rangle \mathrm{d} x \mathrm{~d} t & +c \int_{0}^{\tau}\left\|\rho^{\varepsilon}-r\right\|_{L^{1}(\Omega)}^{2} \mathrm{~d} t \\
& \leq c \int_{0}^{\tau} \mathcal{E}\left(\nu_{t,}^{\varepsilon}, r(t), \mathbf{U}(t)\right) \mathrm{d} t .
\end{aligned}
$$


Further, (1.10) immediately implies

$$
\mathcal{I}_{14} \leq c \int_{0}^{\tau} \mathcal{E}\left(\nu_{t, \cdot}^{\varepsilon}, r(t), \mathbf{U}(t)\right) d t
$$

Finally, by the same method as in $\underline{3}$, Section 5] we derive

$$
\begin{aligned}
\frac{1}{2} \int_{\Omega}\left(\left(\left(\rho^{\varepsilon}-r\right) K *\left(\rho^{\varepsilon}-r\right)\right)(\tau)\right. & \left.-\left(\left(\rho^{\varepsilon}-r\right) K *\left(\rho^{\varepsilon}-r\right)\right)(0)\right) \mathrm{d} x \\
& =\int_{Q_{\tau}}\left\langle\nu_{t, x}^{\varepsilon}, r \mathbf{U}-s \mathbf{v}\right\rangle \nabla_{x} K *\left(r-\rho^{\varepsilon}\right) \mathrm{d} x \mathrm{~d} t,
\end{aligned}
$$

and

$$
\begin{aligned}
& \int_{Q_{\tau}}\left\langle\nu_{t, x}^{\varepsilon}, r \mathbf{U}-s \mathbf{v}\right\rangle \nabla_{x} K *\left(r-\rho^{\varepsilon}\right) \mathrm{d} x \mathrm{~d} t= \\
& \int_{Q_{\tau}}\left(r-\rho^{\varepsilon}\right) \mathbf{U} \nabla_{x} K *\left(r-\rho^{\varepsilon}\right) \mathrm{d} x \mathrm{~d} t+\int_{Q_{\tau}}\left\langle\nu_{t, x}^{\varepsilon}, s(\mathbf{U}-\mathbf{v})\right\rangle \nabla_{x} K *\left(r-\rho^{\varepsilon}\right) \mathrm{d} x \mathrm{~d} t \\
& \leq c \int_{0}^{\tau} \mathcal{E}\left(\nu^{\varepsilon}(t), r(t), \mathbf{U}(t)\right) \mathrm{d} t+c \int_{Q_{\tau}}\left\langle\nu_{t, x}^{\varepsilon}, s|\mathbf{U}-\mathbf{v}|^{2}\right\rangle \mathrm{d} x \mathrm{~d} t \\
& \quad+c\left\|\nabla_{x} K *\left(\rho^{\varepsilon}-r\right)\right\|_{\infty}^{2} \int_{Q_{\tau}} \rho^{\varepsilon} \mathrm{d} x \mathrm{~d} t \leq c \int_{0}^{\tau} \mathcal{E}\left(\nu_{t, \cdot}^{\varepsilon}, r(t), \mathbf{U}(t)\right) d t
\end{aligned}
$$

where we have used the Hölder inequality,

$\int_{(0, \infty) \times \mathbb{R}^{N}} \sqrt{s} \sqrt{s}(\mathbf{U}-\mathbf{v}) \mathrm{d} \nu_{t, x}^{\varepsilon} \leq\left(\int_{(0, \infty) \times \mathbb{R}^{N}} s \mathrm{~d} \nu_{t, x}^{\varepsilon}\right)^{\frac{1}{2}}\left(\int_{(0, \infty) \times \mathbb{R}^{N}} s|\mathbf{U}-\mathbf{v}|^{2} \mathrm{~d} \nu_{t, x}^{\varepsilon}\right)^{\frac{1}{2}}$.

\subsection{Limit}

We collect all the estimates from the previous subsection together with (4.1) in order to derive

$$
\mathcal{E}\left(\nu_{\tau, \cdot}^{\varepsilon}, r(\tau), \mathbf{U}(\tau)\right)+\mathcal{D}^{\varepsilon}(\tau) \leq c \int_{0}^{\tau} \mathcal{E}\left(\nu_{t, \cdot}^{\varepsilon}, r(t), \mathbf{U}(t)\right)+\mathcal{D}^{\varepsilon}(t) \mathrm{d} t+\Gamma(\varepsilon),
$$

for a.a. $\tau \in(0, T)$ and, by the Grönwall's inequality,

$$
\mathcal{E}\left(\nu_{\tau, \cdot}^{\varepsilon}, r(\tau), \mathbf{U}(\tau)\right)+\mathcal{D}^{\varepsilon}(\tau) \leq c \Gamma(\varepsilon),
$$

for a.a. $\tau \in(0, T)$, where $\Gamma(\varepsilon) \rightarrow 0$ as $\varepsilon \rightarrow 0$. This concludes the proof of Theorem 1.4 . 


\section{References}

\section{References}

[1] J. A. Cañizo, J. A. Carrillo, J. Rosado, A well-posedness theory in measures for some kinetic models of collective motion, Math. Models Methods Appl. Sci. 21 (3) (2011) 515-539.

[2] T. Karper, A. Mellet, K. Trivisa, Existence of weak solutions to kinetic flocking models, SIAM J. Math. Anal. 45 (1) (2013) 215-243.

[3] J. A. Carrillo, E. Feireisl, P. Gwiazda, A. Świerczevska-Gwiazda, Weak solutions for Euler systems with non-local interaction, preprint.

[4] E. Chiodaroli, C. D. Lellis, O. Kreml, Global ill-posedness of the isentropic system of gas dynamics, Comm. Pure Appl. Math. 68 (7) (2015) 1157-1190.

[5] E. Chiodaroli, O. Kreml, On the energy dissipation rate of solutions to the compressible isentropic Euler system, Arch. Ration. Mech. Anal. 214 (3) (2014) 1019-1049.

[6] E. Feireisl, O. Kreml, Uniqueness of rarefaction waves in multidimensional compressible Euler system, J. Hyperbolic Differ. Equ. 12 (3) (2015) 489499.

[7] E. Feireisl, P. Gwiazda, A. Świerczevska-Gwiazda, E. Wiedemann, Dissipative measure-valued solutions for the compressible Navier-Stokes system, Calc. Var. Partial Differential Equations 6 (55).

[8] E. Feireisl, Dynamics of viscous compressible fluids, Oxford University Press, Oxford, 2004.

[9] E. Feireisl, B. J. Jin, A. Novotný, Relative entropies, suitable weak solutions, and weak-strong uniqueness for the compressible Navier-Stokes system, J. Math. Fluid Mech. 14 (4) (2012) 717-730. 
[10] A. Novotný, I. Straškraba, Introduction to the Mathematical theorey of Compressible Flow, Oxford Univeristy Press, Oxford, 2004.

[11] P. Pedregal, Parametrized measures and variational principles, Birkhäuser Verlag, Basel, 1997. 